\title{
One-Year Results for Patients with Unilateral Hearing Loss and Accompanying Severe Tinnitus and Hyperacusis Treated with a Cochlear Implant
}

\author{
Angel Ramos Macías ${ }^{a} \quad$ Juan Carlos Falcón-González ${ }^{a}$ \\ Manuel Manrique Rodríguez ${ }^{b}$ Constantino Morera Pérez ${ }^{c} \quad$ Luis García-lbáñez $^{d}$ \\ Carlos Cenjor Español $^{e}$ Chrystelle Coudert-Koall ${ }^{f}$ Matthijs Killian $^{f}$ \\ a Unidad de Hipoacusia, Servicio de Otorrinolaringología, Complejo Hospitalario Universitario Insular Materno

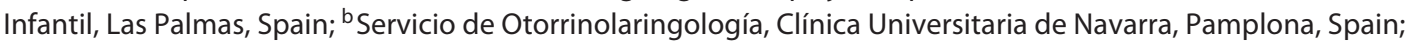 \\ 'Servicio de Otorrinolaringología, Hospital Universitario La Fe, Valencia, Spain; ' Servicio de Otorrinolaringología, \\ Instituto de Otologia Garcia Ibanez, Barcelona, Spain; ' Servicio de Otorrinolaringología, Fundacion Jimenez Diaz,

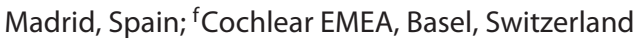

\section{Keywords \\ Cochlear implant - Unilateral hearing loss - Tinnitus . \\ Hyperacusis - Tinnitus Handicap Inventory · Health Utility \\ Index · Speech, Spatial, and Qualities of Hearing Scale}

\begin{abstract}
Objective: To show that patients with unilateral hearing loss $(\mathrm{UHL})$, with one ear fulfilling cochlear implant $(\mathrm{Cl})$ indication criteria, and an additional severe tinnitus handicap can be treated effectively with a Cl. Method: A prospective multicentre study was conducted in five Spanish centres. Sixteen adult patients with UHL and a mean Tinnitus Handicap Inventory (THI) score of at least 58 were implanted. The study design included repeated within-subject measures of quality of life (Health Utility Index Mark 3 [HUI3]), tinnitus (THI, Visual Analogue Scale [VAS] on tinnitus loudness), hearing (Speech, Spatial, and Qualities of Hearing Scale- [SSQ]), and hyperacusis (Test de Hipersensibilidad al Sonido [THS]) up to 12 months after the initial $\mathrm{Cl}$ fitting. Results: Group data showed significant subjective benefit from $\mathrm{Cl}$ treatment: the
\end{abstract}

preoperative HUI3 total utility score of 0.45 went up to 0.57 at 6 months and 0.63 at 12 months; the preoperative THI total score of 75 decreased to 40 at 6 months and 35 at 12 months. The preoperative tinnitus loudness VAS score of 8.2 decreased to 2.4 at 6 months and 2.2 at 12 months with the implant "On" and to 6.7 at 6 months and 6.5 at 12 months with the implant "Off." The preoperative THS total score of 26 decreased to 17 at 12 months. The preoperative SSQ total score of 4.2 increased to 5.1 at 6 months and 6.3 at 12 months. No unanticipated adverse events were reported during the study period. At 12 months after $\mathrm{Cl}$ activation all subjects (except 1 subject who used the device 6 days a week) wore their devices all day and every day. The primary reason for $\mathrm{Cl}$ use was split evenly between tinnitus suppression $(n=6)$ and both hearing and tinnitus $(n=6)$. Conclusion: $\mathrm{A} \mathrm{Cl}$ should be considered as a treatment option in patients with UHL and a concomitant severe tinnitus handicap. However, appropriate counselling of candidates on the anticipated risks, benefits, and limitations that are inherent to cochlear implantation is imperative.

(c) 2018 The Author(s)

Published by S. Karger AG, Basel

\begin{tabular}{ll}
\hline KARGER & $\begin{array}{l}\text { Ko 2018 The Author(s) } \\
\text { Published by S. Karger AG, Basel }\end{array}$ \\
Epenger & apcess \\
E-Mail karger@karger.com & This article is licensed under the Creative Commons Attribution- \\
www.karger.com/aud & $\begin{array}{l}\text { NonCommercial-NoDerivatives 4.0 International License (CC BY- } \\
\text { NC-ND) (http://www.karger.com/Services/OpenAccessLicense). } \\
\text { Usage and distribution for commercial purposes as well as any dis- } \\
\text { tribution of modified material requires written permission. }\end{array}$
\end{tabular}

Matthijs Killian

Cochlear Technology Center

Schaliënhoevedreef 20 I

BE-2800 Mechelen (Belgium)

E-Mail mkillian@ cochlear.com 


\section{Introduction}

Prolonged spontaneous tinnitus of some degree is experienced by up to $20 \%$ of the adult population with exact estimates varying according to the tinnitus definition used [Gopinath et al., 2010; Shargorodsky et al., 2010; Martinez et al., 2015]. Its prevalence is highest among older adults and those with hearing impairment and, in most cases, it is reported as being only mildly annoying [Shargorodsky et al., 2010]. However, for some people their tinnitus is persistent, debilitating, and has a negative impact on their quality of life and speech comprehension, even when present in only one ear [Van de Heyning et al., 2008; Fuiji et al., 2011; Vielsmeier et al., 2016; Weidt et al., 2016]. Tinnitus treatment and assessment is complex with no commonly agreed management pathway and presents an ever-increasing burden on health care services [Martinez et al., 2015; Fuller et al., 2017].

Subjects with tinnitus report poor speech perception, difficulty falling asleep, an inability to concentrate, insecurity, and depression [Tyler and Baker, 1983]. The occurrence of debilitating tinnitus is believed to be associated not only with the dysfunction of the auditory system but with the psychological status and resilience of the sufferer [Dauman and Tyler, 1992; Wallhäusser-Franke et al., 2014; Brüggemann et al., 2016]. Currently, recommended tinnitus treatments focus on improving a patient's ability to ignore and accept the erroneous noise [Tyler et al., 2007]. Techniques such as cognitive behavioural therapy help to alter the noxious response to tinnitus [Cima et al., 2014]. Where patients have a hearing loss, hearing aid fitting is recommended, or if the hearing loss is severe to profound, a cochlear implant (CI) is indicated [Fuller et al., 2017; Zenner et al., 2017]. The prevalence of tinnitus in patients with severe to profound bilateral hearing loss being considered for cochlear implantation is high. Studies report that around $40-50 \%$, or even in excess of $80 \%$, of CI candidates have tinnitus pre-implantation [Quaranta, 2004; Baguley and Atlas, 2007; Kompis et al., 2012; Pierzycki et al., 2016; van Zon, 2016; Mikkelsen, 2017]. After receiving a CI, many patients report that as well as restoring hearing their tinnitus is also reduced, and many studies show significant reductions in tinnitus severity with implant use [Pan et al., 2009; Amoodi et al., 2011; Ramakers et al., 2015; Pierzycki et al., 2016; van Zon, 2016; Bruggeman et al., 2017; Knopke et al., 2017; Zenner et al., 2017]. Initially, it was assumed that this reduction was a consequence of the attention shifting away from the tinnitus towards the environment sounds picked up and transmitted by the CI system, but recent

CI in Patients with Unilateral Hearing

Loss, Tinnitus, and Hyperacusis research has indicated that the tinnitus may be supressed by the intracochlear stimulation, independent of the acoustic input picked up by the sound processor [Arts et al., 2016].

The use of a CI primarily to supress tinnitus has been considered for those patients who have incapacitating tinnitus and a unilateral hearing loss (UHL) and thus would not normally meet the standard criteria for CI. Studies have shown that in this group, the CI has successfully been used to treat the tinnitus symptoms with the benefits lasting long term [Punte et al., 2011; Mertens et al., 2016; Holder et al., 2017]. Uniquely, the CI is also able to restore true binaural input for these patients, not something other unilateral solutions such as bone conduction (Baha) or contralateral routing (CROS) could achieve, providing them with the advantages of binaural hearing for listening in noise and sound localisation [Hoth et al., 2016; Arndt et al., 2017; Dillon et al., 2017; Mertens et al., 2017; Skarzynski et al., 2017]. However, despite the efficacy of providing a CI to patients with UHL and incapacitating tinnitus, funding is not always available. There is a paucity of high-level evidence with many studies reporting on small numbers of patients from single centres. Few studies report generic quality of life measures, an essential requirement in many countries in order to make the case for reimbursement [van Zon et al., 2015; Cabral et al., 2016; Kitterick et al., 2016]. Where quality of life measures have been reported, disease specific measures showed a significant increase, but not in the generic measures [Knopke et al., 2017; Sladen et al., 2017]. However, a very high percentage of patients report wearing their device all day 7 days a week, an important indicator that sufficient benefit is being gained [Friedman et al., 2016; Mertens et al., 2016; Skarzynski et al., 2017].

A link between tinnitus and hyperacusis was noted in a publication by Tyler and Conrad-Armes [1983], and around $40 \%$ of patients with tinnitus also suffer from hyperacusis [Baguley, 2003]. Accumulating evidence suggests that both tinnitus and hyperacusis might share a common cause and be due, in part, to excessive gain increases in the central auditory pathway [Chen et al., 2017; Salvi et al., 2017]. Despite this link between the two conditions, there is little evidence reporting the prevalence of hyperacusis in the implanted population or the impact of a CI.

In this study, the results are reported for a group of patients with a unilateral or asymmetric hearing loss, where one ear fulfils the clinic's standard CI criteria, and an additional severe tinnitus handicap. Unlike other single-centre published studies, patients were recruited from 


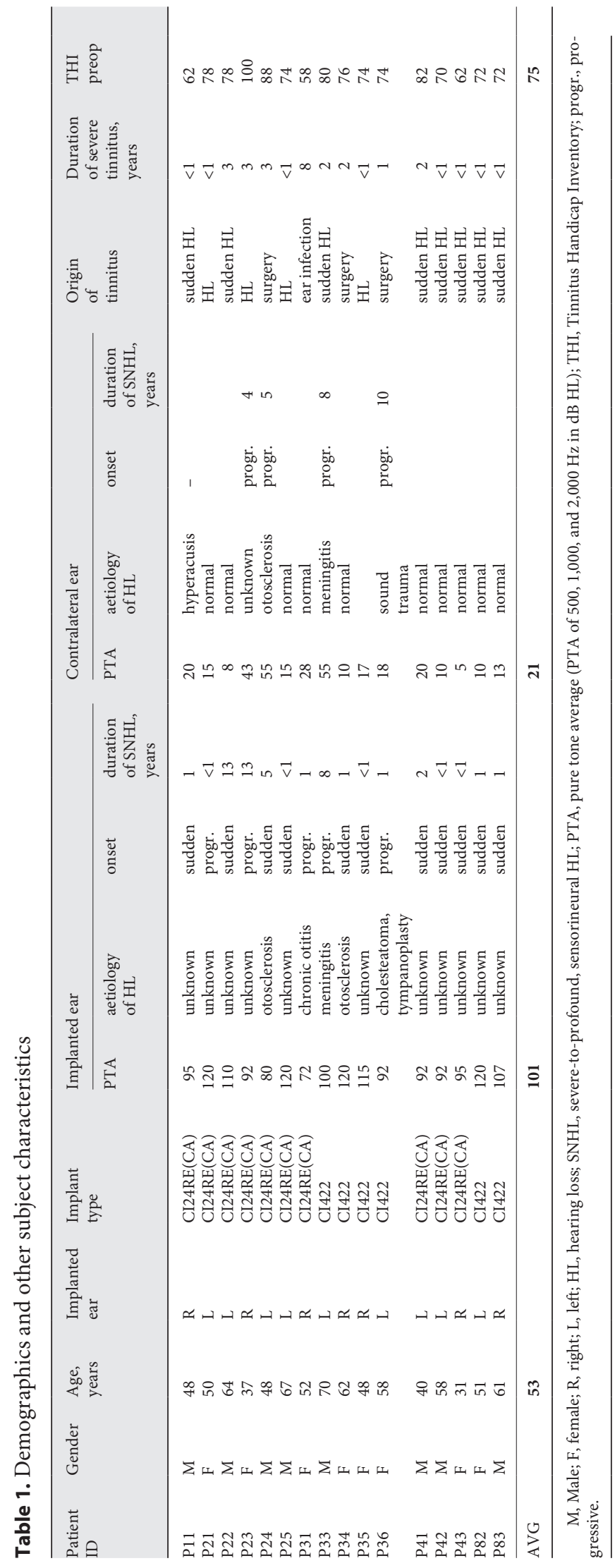

five different Spanish centres, and the focus was on collecting subjective questionnaire data to support reimbursement. The primary outcome measure was active use of the CI. Changes in quality of life were measured using the Health Utilities Mark 3 (HUI3) generic quality of life questionnaire. As well as changes in hearing ability and tinnitus severity, changes in hyperacusis were also assessed. Preliminary results from this study were reported in Ramos Macías et al. [2015], and this paper reports results from the full data set at 12 months after implantation.

The primary objective of this study was to show that patients with UHL, with one ear fulfilling CI indication criteria, and an additional severe tinnitus handicap can be treated effectively with a CI. Treatment was considered to be effective when 12 months after CI activation $\geq 90 \%$ of the patients regularly used their $\mathrm{CI}$ and the group average Tinnitus Handicap Inventory (THI) score showed a statistically significant decrease compared to preoperative scores.

\section{Method}

The study was conducted according to international standards (ISO 14155: 2011). Local ethics committee and National Competent Authority approvals were obtained before the start of the study. Patients were informed by the investigators on the risks and benefits of participating in this study and consented before study enrolment.

\section{Subjects}

Sixteen adult subjects were recruited from multiple test sites. Hearing criteria for inclusion were a hearing loss of less than 15 years' duration in the ear to be implanted, normal hearing or moderate hearing loss (pure tone average $\leq 55 \mathrm{~dB} \mathrm{HL}$ ) in the contralateral ear, and speech perception of less than $50 \%$ on a disyllabic speech test score, in quiet at $65 \mathrm{~dB}$ SPL, in the best aided condition and without lip reading. In addition, subjects were required to have tinnitus caused by or related to the hearing loss, lasting for less than 3 years and with a handicap score greater than 58 , as measured by the THI [Herráiz et al., 2001; McCombe et al., 2001; Newman et al., 1998]. All subjects tried conventional tinnitus treatments including tinnitus retraining therapy for at least 6 months, without a satisfactory result. Exclusion criteria in the ear to be implanted included any cochlear anomaly that might prevent complete insertion of the electrode array and hearing loss related to meningitis, multiple sclerosis, posterior fossa tumours, and of central or retrocochlear origin. Subjects were also excluded if their tinnitus was central in origin (e.g., tumour or cerebrovascular attack), pulsatile, paroxysmal, somato-sensory (e.g., patients report that their tinnitus that can be influenced by jaw or head/neck movements), related to vertigo, headache, or posttraumatic. Subjects with mental health disorders such as complex regional pain syndrome, suicidal thoughts, major depression, or personality disorders (as verified by a psychologist or psychiatrist), or with any additional handicap 
Fig. 1. Audiograms of the study population $(n=16)$. CI-indicated ears: continuous lines and means with diamond symbols. Acoustic hearing ears: dashed lines and means with triangle symbols.

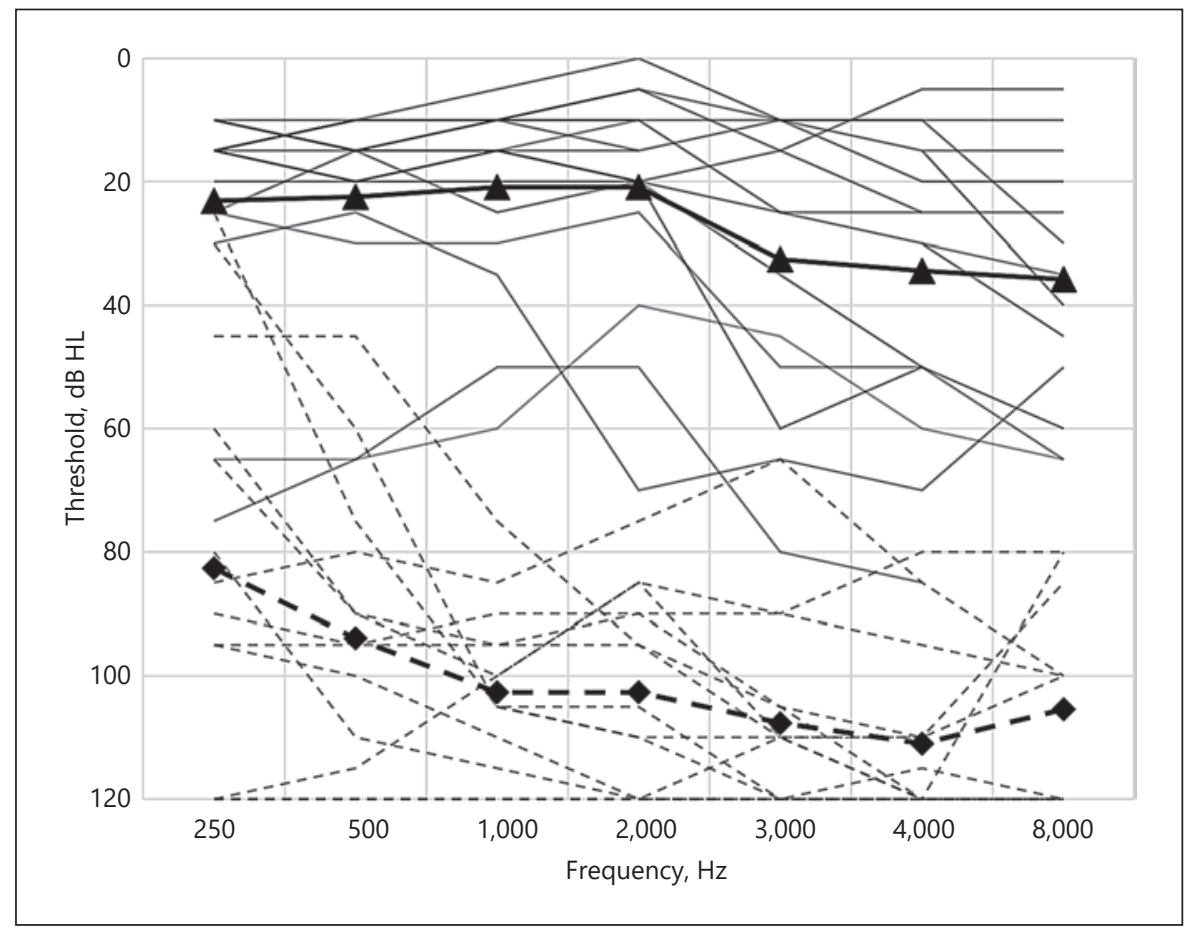

that would prevent participation in evaluations, were also excluded. In addition, patients were excluded with unrealistic expectations regarding the possible benefits, risks, and limitations that are inherent to cochlear implantation.

Table 1 presents the demographics of the study population and details of their hearing loss, tinnitus, and implant type. Mean THI score was 75 (range: 58-100). The preoperative pure tone averages of $500,1,000$, and $2,000 \mathrm{~Hz}$ of the implanted ear ranged from 72 to $120 \mathrm{~dB}$ HL (average: $101 \mathrm{~dB}$, standard deviation [SD]: $15 \mathrm{~dB}$ ) and of the contralateral ear ranged from 5 to $55 \mathrm{~dB}$ HL in the contralateral ear (average: $21 \mathrm{~dB}, \mathrm{SD}: 16 \mathrm{~dB}$ ). The preoperative unaided audiograms are reported in Figure 1. Patient P23, P24, and P33 indicated that they were using a hearing aid in their contralateral ear on a daily basis. In P25, a cochlear ossification was noted during surgery and not all electrodes were inserted into the cochlea.

\section{Procedures}

All subjects attended a preoperative screening and recruitment visit, where baseline measures were made using a battery of questionnaires, and subjects then returned for implant surgery. As part of the CI system, all patients received the CP810 sound processor with a remote assistant. They were fitted with the ACE sound coding strategy using the Custom Sound fitting software and the default parameters used in routine clinical practice. Follow-up assessments were conducted at activation and at 1,6, and 12 months after switch-on.

Tinnitus burden was assessed with the Spanish version of the THI [Newman et al., 1998; Herráiz et al., 2001; McCombe et al., 2001] with the following handicap categories for the THI score: no handicap: 0-16 (grade 1), mild handicap: 18-36 (grade 2), moderate handicap: 38-56 (grade 3), and severe handicap: 58-100 (grade 4 ). The magnitude of the tinnitus loudness/annoyance was as-

CI in Patients with Unilateral Hearing

Loss, Tinnitus, and Hyperacusis sessed with a Visual Analogue Scale (VAS) ranging from 0 to 10 [Miller and Ferris, 1993], with 0 indicating "no tinnitus" and 10 indicating "unbearable tinnitus." In addition, a customised tinnitus questionnaire based on the tinnitus archive [Meikle 1997] was created to fully assess tinnitus characteristics, history, and accompanying symptoms for the study participants. Hyperacusis was assessed with the Sound Hypersensitivity Questionnaire (SHQ), known in Spain as the Test de Hipersensibilidad al Sonido (THS) [Herráiz et al., 2006], which was based on the German "Geräuschüberempfindlichkeit" questionnaire [Nelting et al., 2002]. The THS handicap categories are as follows: mild: 1-10 (grade I), moderate: 11-17 (grade II), severe: 18-25 (grade III), and very severe: 26-45 (grade IV). Quality of life was assessed with the HUI3 (http://www.healthutilities.com/) and quality of hearing ability was assessed with the Speech, Spatial, and Qualities of Hearing Scale (SSQ) [Gatehouse and Noble, 2004].

Subjects had to fill in a paper version of the questionnaires before they came to each study visit and clinicians checked each questionnaire to ensure they were fully completed.

\section{Statistical Analysis}

Pre- and postoperative questionnaire results were compared using both parametric and non-parametric statistics. The distribution of the outcome values across the various visits along with the difference compared to baseline was examined using Q-Q plots and further evaluated using Shapiro-Wilk tests for normal distribution. Both the non-parametric Wilcoxon signed-rank test (2-sided) and paired $t$ tests were used to examine differences from baseline to month 12 for the various outcome variables (i.e., THI, THS, and SSQ scores including the subscales). Parametric and non-parametric tests yield consistent findings and the $t$ test results are reported. No adjustment of the type I error for multiple testing 


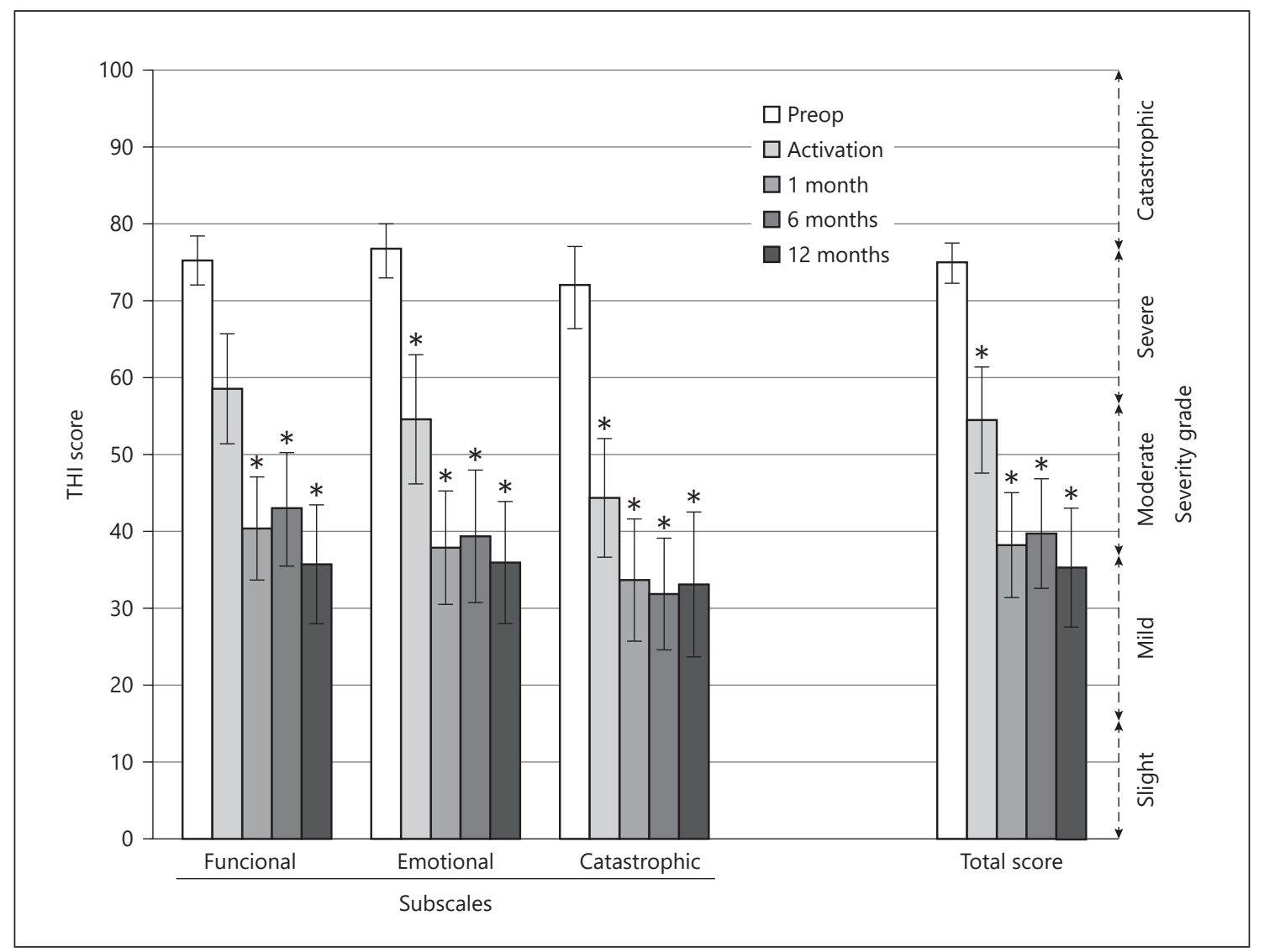

Fig. 2. Tinnitus Handicap Inventory (THI) total and subscale scores (mean \pm standard error of mean, $n=16$ ). * Indicates a significant difference with respect to preoperative scores.

Table 2. Hours of CI use and primary reasons for continuing to wear the CI

\begin{tabular}{|c|c|c|c|c|c|c|c|c|c|}
\hline \multirow{2}{*}{$\begin{array}{l}\text { Patient } \\
\text { ID }\end{array}$} & \multicolumn{3}{|c|}{ Use, days/week } & \multicolumn{3}{|c|}{ Use, hours/day } & \multicolumn{3}{|c|}{ Use (hearing/tinnitus/both) } \\
\hline & $1 \mathrm{M}$ & $6 \mathrm{M}$ & $12 \mathrm{M}$ & $1 \mathrm{M}$ & $6 \mathrm{M}$ & $12 \mathrm{M}$ & $1 \mathrm{M}$ & $6 \mathrm{M}$ & $12 \mathrm{M}$ \\
\hline P11 & 7 & 7 & 7 & 6 & 8 & 10 & both & tinnitus & tinnitus \\
\hline P21 & 7 & 7 & 7 & 8 & 9 & 6 & tinnitus & tinnitus & tinnitus \\
\hline P22 & 7 & 7 & 7 & 10 & 9 & 9 & both & both & both \\
\hline P23 & 7 & 7 & 7 & 19 & 8 & 10 & tinnitus & tinnitus & tinnitus \\
\hline P24 & 7 & 7 & 7 & 10 & 10 & 10 & tinnitus & tinnitus & tinnitus \\
\hline P25 & 7 & 7 & 7 & 7 & 9 & 10 & tinnitus & tinnitus & tinnitus \\
\hline P31 & 7 & 7 & 7 & 16 & 16 & 16 & both & both & tinnitus \\
\hline P33 & 7 & 7 & 7 & 14 & 14 & 16 & tinnitus & tinnitus & both \\
\hline P34 & 7 & 7 & 7 & 12 & 12 & 12 & hearing & both & both \\
\hline P35 & 7 & 7 & & & 16 & & & both & both \\
\hline P36 & 7 & 7 & 7 & & 15 & 12 & & both & both \\
\hline P41 & 7 & 7 & 7 & 14 & 14 & 10 & tinnitus & both & tinnitus \\
\hline P42 & 7 & 7 & 7 & 14 & 17 & 15 & tinnitus & tinnitus & tinnitus \\
\hline $\mathrm{P} 43$ & 7 & 7 & 6 & 14 & 12 & 12 & tinnitus & both & both \\
\hline P82 & 7 & 7 & 7 & 12 & 16 & 12 & both & both & both \\
\hline P83 & 7 & 7 & 7 & 12 & 10 & 11 & both & both & both \\
\hline
\end{tabular}

CI, cochlear implant; $\mathrm{M}$, month. 
was carried out since all tests were considered of an explorative nature. $p$ values less than 0.05 were deemed as statistically significant. The SAS software Version 9.4 was used for statistical analysis (SAS Institute, Cary, NC, USA).

\section{Results}

\section{Tinnitus}

Patients reported their tinnitus to be located in or around their profoundly deaf ear and this did not change over the study period of 12 months. Patients described their tinnitus sounds over time based on pre-defined descriptions, with an opportunity to add unlisted sounds. The tinnitus sounds heard most were hissing, buzzing, and transformer noise. Tonal tinnitus was rare. Reports on the types of sounds and the loudness of sounds tended to be stable over time and variations in pitch/loudness were reported to be related to implantation, stress, fatigue, noise exposure, or shift from day to night.

Information on previous tinnitus therapies was available in 14 subjects. Masking was tried in 2 patients (it was ineffective in one and in the other no further data on effectiveness were available. Counselling was provided in 4 patients but offered no relief. In four patients, tinnitus retraining therapy was provided in 4 patients (it provided relief from tinnitus in 1 patient and was ineffective in 3 patients). Acupuncture was tried in 3 patients (in 2 patients it did not provide relief and in 1 patient no further data were available). One patient tried "autocontrol," an unspecified self-control technique, for tinnitus relief. Nine patients tried several drugs to find relief from tinnitus (in 6 patients this did not provide relief and in 2 patients there were no further data on their effectiveness).

At 12 months after surgery, 14 out of 16 subjects continued to wear their CI every day. One subject wore their device 6 days a week and for 1 subject only 6-month data were available. The average number of hours of daily use was $12 \mathrm{~h}$ at 1 and 6 months and $11 \mathrm{~h}$ at 12 months (range: 6-16 h). Eight subjects indicated that the primary reason for implant use was tinnitus suppression and 8 subjects indicated that it was for better hearing and tinnitus suppression (Table 2).

Residual inhibition was measured after presentation of a 1-min electrical or acoustical stimulus at the planned study visit. For these measurements the electrical stimulus was identified by matching stimulation on five sequential electrodes to the dominant tinnitus pitch and then identifying the minimal masking level (MML), i.e., the lowest level at which tinnitus was fully masked. The

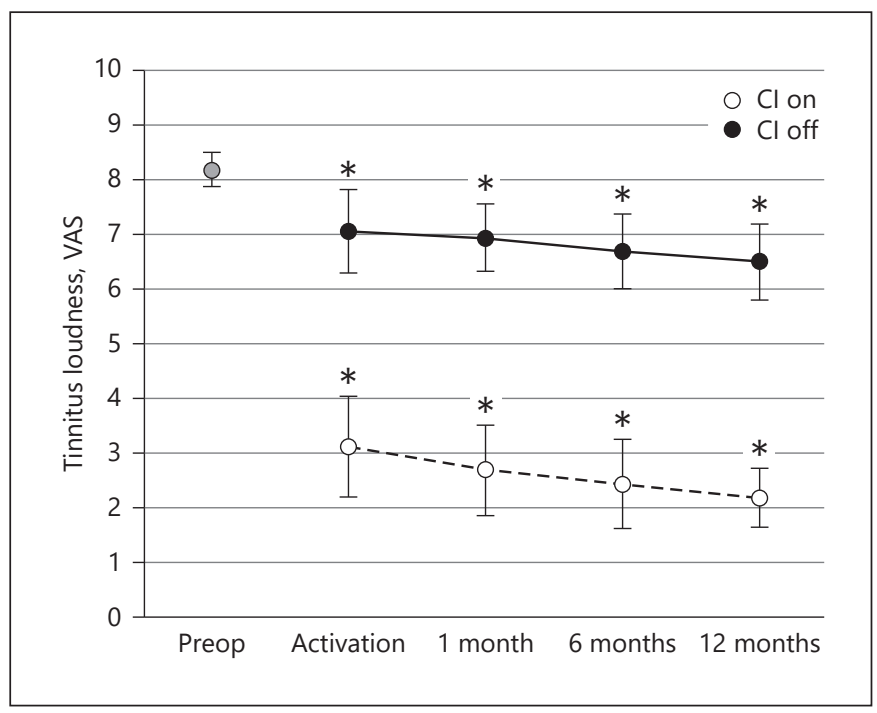

Fig. 3. Tinnitus loudness scores (mean \pm standard error of mean, $n=16)$. *Indicates a significant difference with respect to preoperative scores. Scores were obtained with numerical Visual Analogue Scale (VAS; range 0-10: $0=$ no tinnitus, $10=$ unbearably loud tinnitus).

acoustic stimulus was identified by matching a narrow band noise to the tinnitus pitch, identifying the MML, and then setting the stimulus to MML $+10 \mathrm{~dB}$. The dataset was not complete; electrically a total of 31 observations were logged in 11 patients and acoustically a total of 57 observations were logged in 16 patients. The median, dominant, pitch-matched electrode in the centre of the five sequential electrodes was EL13 (range: EL5 to EL22). The electrical MML ranged between 2 and 77 currentlevel units above the electrical hearing threshold level. The median, dominant acoustic centre frequency for the narrow noise band was $1,500 \mathrm{~Hz}$ (range: $125 \mathrm{~Hz}$ to $8 \mathrm{kHz}$ ). The acoustic MML ranged from 0 - to $85 \mathrm{~dB}$ HL and could not always be measured due to hyperacusis. Residual inhibition varied from $0 \mathrm{~s}$ to more than $5 \mathrm{~min}$ and was generally short lasting with a median of 30-60 s for the electrical stimulation and a median of 10-30 s for the acoustic stimulation. It did not show a general trend towards change over the study time period of 12 months.

At 12 months after activation, THI scores decreased by 2 grades from catastrophic/severe to moderate/mild and the decrease in scores was statistically significantly compared to preoperative values in all domains (total: $t=$ $-5.79, p<0.0001$; functional: $t=-4.19, p=0.0002$; emotional: $t=-6.95 p<0.0001$; catastrophic: $t=-4.92, p=$ 0.0002 ) (Fig. 2). After an initial improvement at activation and at 1 month, the scores plateaued. 
Fig. 4. Test for hypersensitivity to sound (Test de Hipersensibilidad al Sonido [THS]) total and subscale scores (mean \pm standard error of mean, $n=16)$. ${ }^{*}$ Indicates a significant difference with respect to preoperative scores. Subjects rated 15 questions according to never, frequently, always, or on occasion.

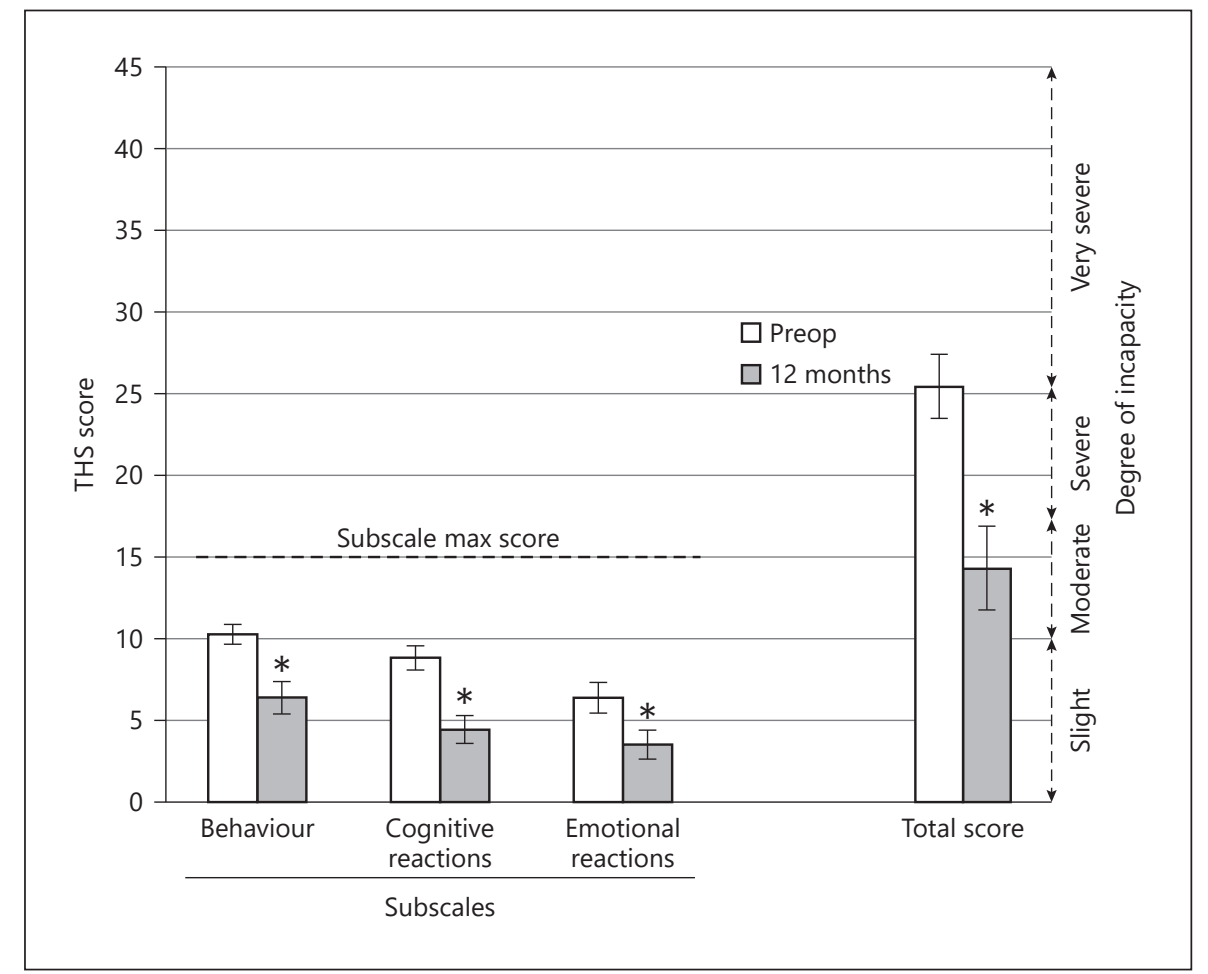

In addition to the THI questionnaire, patients were asked to complete a VAS on tinnitus loudness preoperatively, at activation, and at 1,6 , and 12 months with the implant "On" and "Off" (Fig. 3). At 12 months after activation, scores decreased significantly compared to preoperative values after cochlear implantation by $5-6$ points with the CI activated (CI On) $(t=-0.82, p<0.0001)$ and by $1-2$ points when the CI was inactive (CI Off) $(t=-2.3$, $p<0.05)$.

\section{Hearing}

At 12 months after activation, the THS total score and the score on the cognitive reactions, actional/somatic behaviour, and emotional reaction to external noises subscales were all significantly lower than the preoperative scores (Fig. 4) (total: $t=-3.61, p=0.0026$; behaviour: $t=$ $-3.06, p=0.008$; cognitive: $t=-4.21, p=<0.001$; emotional: $t=-2.38, p=0.031$ ). Clinically, ratings dropped from very severe to moderate. Preoperative scores were high, indicating substantial interference of tinnitus/hyperacusis with hearing.

In the SSQ, total and subdomain scores significantly improved compared to preoperative scores at 12 months after CI activation (total: $t=6.77, p<0.0001$; speech: $t=$ 6.65, $p<0.0001$; spatial: $t=4.65, p<0.001$; qualities: $t=$
$6.1, p<0.0001)$ and the speech subdomain score also showed a significant improvement 6 months after CI activation $(t=3.13, p<0.01)$ (Fig. 5).

\section{Quality of Life}

The HUI3 total utility score significant improved 12 months after activation from 0.45 to 0.63 , a gain of 0.18 $(t=2.63, p<0.01)$. Both the hearing domain $(t=2.49$, $p=0.03)$ and the emotion domain $(t=2.13, p=0.05)$ showed a significant improvement 12 months after activation. There were no significant differences at 6 months after activation (Fig. 6).

\section{Discussion}

The study met its primary aims with over $90 \%$ of subjects wearing their device 6-7 days a week at 12 months after activation. Half of the subjects indicated that their main reason for using the $\mathrm{CI}$ was for tinnitus suppression and the other half for both hearing and tinnitus suppression. This is consistent with Mertens et al. [2016], who reported tinnitus suppression as the primary benefit in $83 \%$ of the patients with single-sided deafness. This primary benefit shifted to improved hearing, when the hear- 
Fig. 5. Speech, Spatial and Qualities of Hearing Scale (SSQ) total and subscale scores (mean \pm standard error of mean, $n=$ 16). * Indicates a significant difference with respect to preoperative scores. Scores were obtained on a 10 -point Likert Scale $(0=$ not at all, $10=$ perfectly).
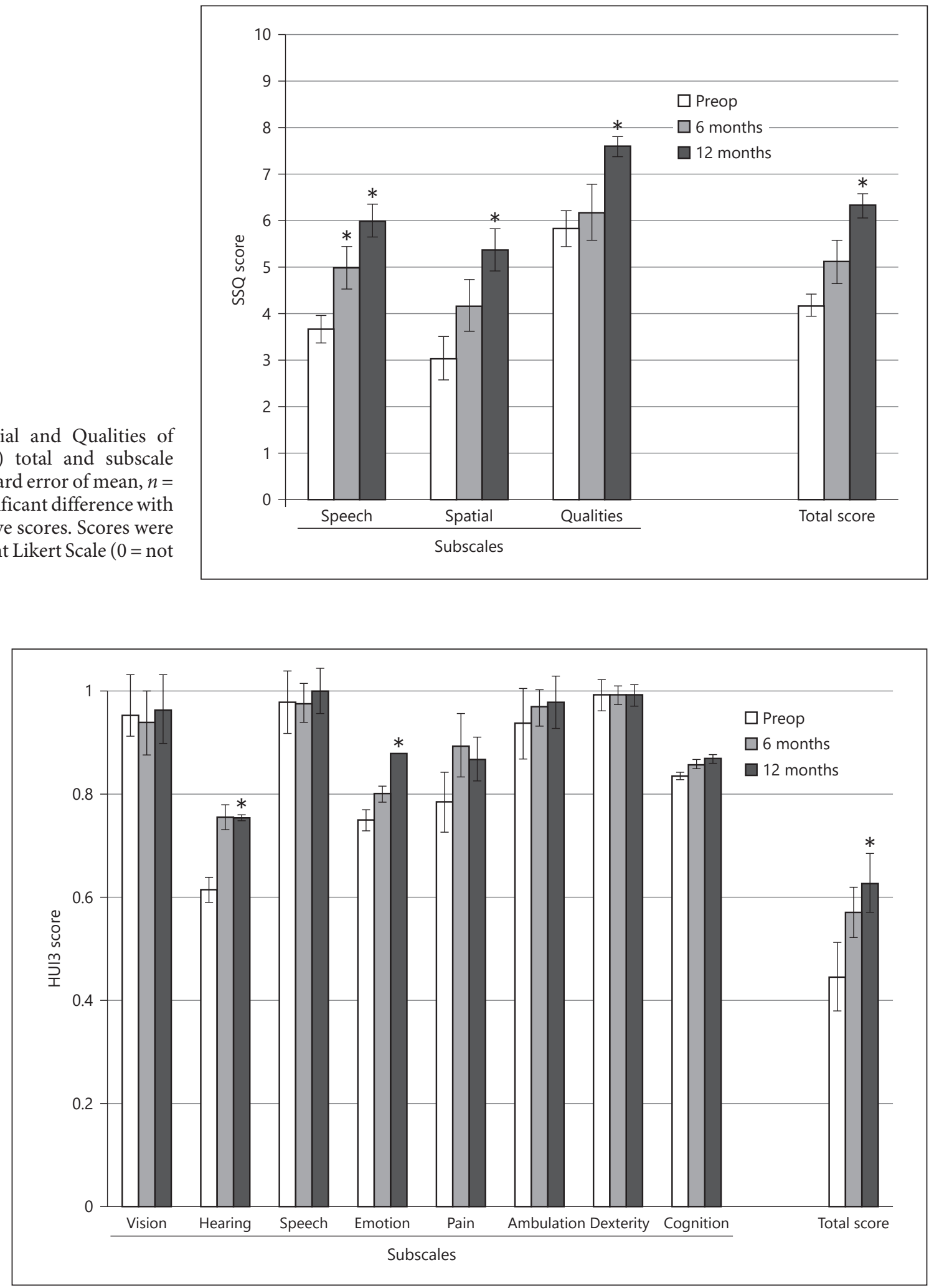

Fig. 6. Health Utility Index Mark 3 (HUI3) total and subscale scores (mean \pm standard error of mean, $n=16$ ). * Indicates a significant difference with respect to preoperative scores. A score of 1 represents perfect health and a score of 0 represents dead. 
ing loss in the acoustic hearing ear became worse and patients were considered to have asymmetric hearing loss. Other studies have also shown high levels of long-term CI use in this UHL population, and this is a good indicator that sufficient benefit is being provided [Friedmann et al., 2016; Mertens et al 2016; Skarzynski et al., 2017]. The results also compare very favourably with the non-use rates of $13-14 \%$ reported for alternative treatment options such as bone-anchored hearing aids [Mertens et al., 2017]. This is an important finding as when the cost-effectiveness of a treatment is evaluated non-use can have a large negative impact on the final calculation.

The impact of cochlear implantation on tinnitus reduction is well established [Amoodi et al., 2011; Bruggeman et al., 2017; Knopke et al., 2017; Pan et al., 2009; Pierzycki et al., 2016; Ramakers et al., 2015; van Zon, 2016; Zenner et al., 2017]. The results reported here also show both a statistically and clinically significant reduction in tinnitus loudness and handicap. The subjects recruited had a high level of tinnitus handicap before implantation, categorised as catastrophic to severe, and this was reduced to mild after implantation. Most of this reduction occurred in the first month following implantation and THI scores then plateaued. The THI has been used in two other studies of subjects with UHL, and although the initial tinnitus handicap levels of the samples before implantation were lower than in this study at moderate to severe, the final recorded postoperative scores were also categorised as mild [Arts et al., 2016; Holder et al., 2017]. Tinnitus loudness was high in this sample before implantation (loudness VAS: 8/10) and continued to be significantly reduced at 12 months after activation (loudness VAS: 2/10). Long-term studies reporting results up to 10 years after activation also show continued reduction of tinnitus loudness to very low levels and 100\% continued device use [Mertens et al., 2016]. A significant long-term reduction in tinnitus loudness scores was also observed with the implant off, although the effect was much smaller than with the implant on. The same effect was observed by Mertens et al. [2016] in 2 subjects and may be a placebo effect whereby the psychological status of the sufferer is improved, either by better hearing or access to a new and expensive treatment, and thus tinnitus is reduced [Brüggemann et al., 2016]. An alternative hypothesis is that routine daily use of the implant leads to residual tinnitus inhibition with prolonged time constants. In some patients this residual inhibition could last overnight (i.e., the switch-off period) and occasionally provides full tinnitus inhibition during day and night as reported regularly in studies with conventional CI candidates with less burdensome tinnitus [Baguley and Atlas, 2007].

In the study by Mertens et al. [2016], residual inhibition of tinnitus after switching off the CI was generally less than $10 \mathrm{~min}$, even after several years of use [Mertens et al., 2016]. In the current study, residual inhibition was short lasting with a median of less than $1 \mathrm{~min}$. These results suggest that long-term CI use is not able to reverse the maladapted tinnitus brain [Moller, 2000; Eggermont, 2007; Knipper et al., 2013]. The active implant provides meaningful input which helps the patients to reduce their ever-present tinnitus percept, which is in line with the observation that it was a complex clinical operation to identify a reliable and stable MML in this study. Furthermore, CI users reported that they still heard their tinnitus for about $50 \%$ of the time during the day, even with the CI active.

Overall, there was a significant subjective benefit to hearing and localisation as measured by the SSQ questionnaire. Half the subjects reported using the device for both hearing and tinnitus, and SSQ scores were highly statistically significant for all subdomains, including qualities of hearing. The contribution to improved binaural hearing that the implant provides is also reflected in the significant improvement in scores for the hearing domain of the HUI3.

A unique aspect of this study was the inclusion of the THS hyperacusis measure. Gathering this data is especially relevant when the need for treatment is even greater in patients with tinnitus and hyperacusis. Schecklmann et al. [2014] showed that the ability to ignore tinnitus was subjectively decreased in hyperacusis patients, and they suffered from increased depression and decreased quality of life compared to those with tinnitus alone.

The Hyperacusis Handicap score decreased significantly from 26 preoperatively to 17 at 12 months, which represented a change from severe/very severe incapacity before implantation to moderate incapacity with 12 months of implant use. Mertens et al. [2016] presented results for the Hyperacusis Questionnaire in their 10-year follow-up study of patients with UHL implanted with a $\mathrm{CI}$, and were able to demonstrate a reduction in scores with the CI on. They found, however, that the CI only made a significant difference in the group with singlesided deafness, not the asymmetric hearing loss group. However, the questionnaire they used was focused on problems with hearing due to the hyperacusis, unlike the questionnaire used here which was more focused on the impact of loud sounds and noise on quality of life and thus may be more sensitive to hyperacusis changes. In the
16

Audiol Neurotol 2018;23:8-19 DOI: $10.1159 / 000488755$
Ramos Macías et al. 
validation study of the THS, 90\% percent of patients (36 cases) also complained of tinnitus, and it was found that tinnitus scores on the VAS and the THI were positively correlated to higher THS scores. This supports the theory that the two conditions may have a common cause [Herráiz et al., 2006]. However, a principal component analysis on the available dataset, including the THI, THS, and SSQ total and subdomain scores, did not reveal a strong internal structure in the dataset as the decay in the explained variance was quite smooth, and the first principal component analysis accounted for $45 \%$ of the variance.

For the first time in this patient population a significant improvement in health utility after implantation was shown. Arndt et al. [2017] reported on a large series of patients from their clinic. They showed that a CI was a possibility for the rehabilitation of patients with singlesided deafness and provided superior performance for speech comprehension in noise and localisation compared to other treatment options. However, no measures of quality of life or tinnitus assessments were included. A prospective, randomised, controlled, single-centre trial of treatment options for single-sided deafness is underway which will also compare the efficacy of CROS, bone conduction, and CI and includes a comprehensive battery of quality of life measures and tinnitus questionnaires; however, results are yet to be reported [Peters et al., 2015]. The HUI3 was chosen as the generic quality of life measure over the alternatives such as the Euro-QOL and SF-36, as it is regarded as the most sensitive of these measures to hearing difficulties and specifically tinnitus [Maes et al., 2011; Ramakers et al., 2016]. Other studies have shown a significant quality of life benefit with disease-specific measures, which are even more sensitive than the HUI3, but have been unable to show a benefit using a generic quality of life questionnaire, which is required by many funding bodies. Both Arts et al. [2016] and Arndt et al. [2010] used the HUI3 and recorded improvements in health utility but were unable to show a statistically significant gain. Arndt et al. [2010] did, however, show a significant improvement in the HUI3 overall score for the CI compared to CROS hearing aid treatment (utility gain of 0.15 ) and Baha treatment (utility gain of 0.13 ). This study showed a statistically and clinically significant overall utility gain of 0.18 on the HUI3. Differences in HUI3 scores came from the hearing domain, as expected, but also from the emotion domain. The emotion questions asked if subjects were happy and interested in life and ranged down to very unhappy or so unhappy life is not worth living. The large utility gain of 0.18 recoded is equivalent to the gains recorded in a multinational survey

CI in Patients with Unilateral Hearing

Loss, Tinnitus, and Hyperacusis of 291 bilaterally deafened but unilaterally implanted traditional CI patients, including patients with tinnitus and children over 10 years of age [Lenarz et al., 2017]. If we consider the baseline health status of the two groups, both had a utility score of 0.45 . This is lower than the utility scores recorded for tinnitus patients undergoing conventional treatment. Maes et al. [2011] reported mean utility scores of a large sample for those with severe tinnitus but no mild hearing loss of 0.55 and an average utility across all 428 tinnitus patients of 0.64 [Maes et al., 2011]. These results indicate that patients with tinnitus and UHL feel considerably worse off than those with tinnitus alone and similarly disadvantaged to more traditional implant candidates with bilateral hearing loss. The size of the health utility gains shown here demonstrate that the impact on quality of life of the CI on these two groups is equivalent and a CI should be considered as an effective treatment for this population.

The study is limited by the fact that no control group was included to evaluate alternative treatment options such as Baha or CROS. The sample was also highly selective with patients included who had a high degree of tinnitus handicap and low health utility scores. Future research should aim to replicate these results in a larger sample with a control group and to explore further the contributions of improved hearing and reduced tinnitus to the improvements seen in quality of life.

\section{Conclusions}

The primary objective was met and all subjects (except 1 subject who used the device 6 days a week) wore their devices all day and every day. Half of the patients indicated tinnitus as the primary reason for CI use and half indicated they used their CI for both hearing and tinnitus. Tinnitus loudness (VAS) and handicap (THI) decreased both clinically and statistically significantly during active use of the implant, with the preoperative tinnitus loudness VAS of 8.2 decreasing to 2.2 at 12 months and the preoperative THI of 75 decreasing to 35 at 12 months. Subjective hearing improved significantly as shown by the SSQ, increasing from a preoperative score of 4.2 to 6.3 at 12 months, and the hearing domain in the HUI3, increasing from a preoperative score of 0.61 to 0.75 at 12 months. Hyperacusis handicap (THS) decreased significantly from a preoperative score of 26 to 17 at 12 months. Generic quality of life improved significantly with a 0.18 improvement (from a preoperative score of 0.45 to 0.63 at 12 months) on the HUI3, comparable to utility gains 
seen in traditional CI users with binaural hearing loss. Patients with UHL loss with indications for a CI and a concomitant severe tinnitus handicap were successfully treated with a CI. A CI should be considered as a viable and cost-effective treatment option for this population. However, the sample was highly selective and careful patient selection for this treatment is imperative.

\section{Acknowledgements}

The authors would like to thank Dr. Silvia Borkoski Barreiro (Las Palmas), Dr. Laura Cavalle Garrido (Valencia), Dr. Alicia Huarte Irujo (Pamplona), Pilar Martinez Barbarin (Pamplona), Charo Lezaun Alfonso (Pamplona), Elisabeth Estrada Leypon
(Barcelona), Sandra Salinas (Madrid), and Gabriela Mecco López (Madrid) for their support with the execution of the study. They would also like to thank Dr. Ernst von Wallenberg (Cochlear Company) for his help with the conception of the study. In addition, they wish to thank Dr. Dominik H. Pfluger (Numerics Data $\mathrm{GmbH}$ ) for providing independent statistical advice, Ms. Paula Greenham (Greenham Research Consulting) for her help in the writing of the manuscript, and the anonymous reviewers who helped to improve the paper.

\section{Disclosure Statement}

Matthijs Killian and Chrystelle Coudert-Koall are employees of the Cochlear Company. No further conflict of interest is reported by the authors.

\section{References}

Amoodi HA, Mick PT, Shipp DB, Friesen LM, Nedzelski JM, Chen JM Lin VY: The effects of unilateral cochlear implantation on the tinnitus handicap inventory and the influence on quality of life. Laryngoscope 2011;121: 1536-1540.

Arndt S, Aschendorff A, Laszig R, Beck R, Schild C, Kroeger S, Ihorst G, Wesarg T: Comparison of pseudobinaural hearing to real binaural hearing rehabilitation after cochlear implantation in patients with unilateral deafness and tinnitus. Otol Neurotol 2010; 32:39-47.

Arndt S, Laszig R, Aschendorff A, Hassepass F, Beck R, Wesarg T: Cochlear implant treatment of patients with single-sided deafness or asymmetric hearing loss. HNO 2017;10:1-12.

Arts RA, George EL, Janssen M, Griessner A, Zierhofer C, Stokroos RJ: Tinnitus suppression by intracochlear electrical stimulation in single sided deafness - a prospective clinical trial: follow-up. PLoS One 2016;11:e0153131.

Baguley DM: Hyperacusis. J R Soc Med 2003;96: 582-585.

Baguley DM, Atlas MD: Cochlear implants and tinnitus. Prog Brain Res 2007;166:347-355.

Brüggemann P, Szczepek AJ, Rose M, McKenna L, Olze H, Mazurek B: Impact of multiple factors on the degree of tinnitus distress. Front Hum Neurosci 2016;10:341.

Cabral F Jr, Pinna MH, Alves RD, Malerbi AF, Bento RF: Cochlear implantation and singlesided deafness: a systematic review of the literature. Int Arch Otorhinolaryngol 2016;20: 69-75.

Chen YC, Chen GD, Auerbach BD, Manohar S, Radziwon K, Salvi R: Tinnitus and hyperacusis: contributions of paraflocculus, reticular formation and stress. Hear Res 2017;349:208222
Cima RF, Andersson G, Schmidt CJ, Henry JA: Cognitive-behavioral treatments for tinnitus: a review of the literature; J Am Acad Audiol 2014;25:29-61.

Dauman R, Tyler RS: Some considerations on the classification of tinnitus; in Aran JM, Dauman R (eds): Tinnitus 91: Proceedings of the Fourth International Tinnitus Seminar. Amsterdam, Kugler, 1992, pp 225-229.

Dillon MT, Buss E, Anderson ML, King ER, Deres EJ, Buchman CA, Brown KD, Pillsbury HC: Cochlear implantation in cases of unilateral hearing loss: initial localization abilities. Ear Hear 2017;38:611-619.

Eggermont JJ: Pathophysiology of tinnitus. Prog Brain Res 2007;166:19-35.

Friedmann DR, Ahmed OH, McMenomey SO, Shapiro WH, Waltzman SB, Roland JT Jr: Single-sided deafness cochlear implantation: candidacy, evaluation, and outcomes in children and adults. Otol Neurotol 2016;37:e154e160.

Fujii K, Nagata C, Nakamura K, Kawachi T, Takatsuka N, Oba S, Shimizu H: Prevalence of tinnitus in community-dwelling Japanese adults. J Epidemiol 2011;21:299-304.

Fuller TE, Haider HF, Kikidis D, Lapira A, Mazurek B, Norena A, Rabau S, Lardinois R, Cederroth CR, Edvall NK, Brüggemann PG, Rosing SN, Kapandais A, Lungaard D, Hoare DJ, Cima RF: Different teams, same conclusions? A systematic review of existing clinical guidelines for the assessment and treatment of tinnitus in adults. Front Psychol 2017;22:8-206.

Gatehouse S, Noble W: The Speech, Spatial and Qualities of Hearing Scale (SSQ). Int J Audiol 2004;43:85-99.

Gopinath B, McMahon CM, Rochtchina E, Karpa MJ, Mitchell P: Incidence, persistence, and progression of tinnitus symptoms in older adults: the Blue Mountains Hearing Study. Ear Hear 2010;31:407-412.
Herráiz C, de los Santos G, Diges I, Díez R, Aparicio JM: Assessment of hyperacusis: the selfrating questionnaire on hypersensitivity to sound (in Spanish). Acta Otorrinolaringol Esp 2006;57:303-306.

Holder JT, O'Connell B, Hedley-Williams A, Wanna G: Cochlear implantation for singlesided deafness and tinnitus suppression. Am J Otolaryngol 2017;38:226-229.

Hoth S, Rösli-Khabas M, Herisanu, Plinkert PK, Praetorius M: Cochlear implantation in recipients with single-sided deafness: audiological performance. Cochlear Implants Int 2016;17:190-199.

Kitterick PT, Smith SN, Lucas L: Hearing instruments for unilateral severe-to-profound sensorineural hearing loss in adults: a systematic review and meta-analysis. Ear Hear 2016;37: 495-507.

Knipper M, Van Dijk P, Nunes I, Rüttiger L, Zimmermann U: Advances in the neurobiology of hearing disorders: recent developments regarding the basis of tinnitus and hyperacusis. Prog Neurobiol 2013;111:17-33.

Knopke S, Szczepek AJ, Häussler SM, Gräbel S, Olze H: Cochlear implantation of bilaterally deafened patients with tinnitus induces sustained decrease of tinnitus-related distress. Front Neurol 2017;8:185.

Kompis M, Pelizzone M, Dillier N, Allum J, DeMin N, Senn P: Tinnitus before and 6 months after cochlear implantation. Audiol Neurootol 2012;17:161-168.

Lenarz T, Muller L, Czerniejewska-Wolska H, Vallés Varela H, Orús Dotú C, Durko M, Huarte Irujo A, Piszczatowski B, Zadrożniak M, Irwin C, Graham PL, Wyss J: Patient-related benefits for adults with cochlear implantation: a multicultural longitudinal observational study. Audiol Neurootol 2017;22:6173. 
Maes IH, Joore MA, Cima RF, Vlaeyen JW, Anteunis LJ: Assessment of health state in patients with tinnitus: a comparison of the EQ5D and HUI mark III. Ear Hear 2011;32:428435.

Martinez C, Wallenhorst C, McFerran D, Hall D: Incidence rates of clinically significant tinnitus: 10-year trend from a cohort study in England. Ear Hear 2015;36:e69-e75.

McCombe A, Baguley D, Coles R, McKenna L, McKinney C, Windle-Taylor P; British Association of Otolaryngologists, Head and Neck Surgeons: Guidelines for the grading of tinnitus severity: the results of a working group commissioned by the British Association of Otolaryngologists, Head and Neck Surgeons, 1999. Clin Otolaryngol Allied Sci 2001;26: 388-393.

Meikle MB: Electronic access to tinnitus data: the Oregon Tinnitus Data Archive. Otolaryngol Head Neck Surg 1997;117:698-700.

Mertens G, De Bodt M, Van de Heyning P: Evaluation of long-term cochlear implant use in subjects with acquired unilateral profound hearing loss: focus on binaural auditory outcomes. Ear Hear 2017;38:117-125.

Mertens G, De Bodt M, Van de Heyning P: Cochlear implantation as a long-term treatment for ipsilateral incapacitating tinnitus in subjects with unilateral hearing loss up to 10 years. Hear Res 2016;331:1-6.

Mikkelsen KS, Ovesen T, Swan CZ: Pre- and postoperative dizziness, tinnitus, and taste disturbances among cochlear implant recipients. J Laryngol Otol 2017;131:309-315.

Miller MD, Ferris DG: Measurement of subjective phenomena in primary care research: the $\mathrm{Vi}$ sual Analogue Scale. Fam Pract Res J 1993;13: $15-24$.

Moller AR: Similarities between severe tinnitus and chronic pain. J Am Acad Audiol 2000;11: 115-124.

Nelting M, Rienhoff NK, Hesse G, Lamparter U: The assessment of subjective distress related to hyperacusis with a self-rating questionnaire on hypersensitivity to sound (in German). Laryngorhinootologie 2002;81:327334.

Newman CW, Sandridge SA, Jacobson GP: Psychometric adequacy of the Tinnitus Handicap Inventory (THI) for evaluating treatment outcome. J Am Acad Audiol 1998;9:153-160.

Pan T, Tyler RS, Ji H, Coelho C, Gehringer AK, Gogel SA: Changes in the tinnitus handicap questionnaire after cochlear implantation. Am J Audiol 2009;18:144-151.
Peters JP, van Zon A, Smit AL, van Zanten GA, de Wit GA, Stegeman I, Grolman W: CINGLEtrial: cochlear implantation for siNGLE-sided deafness, a randomised controlled trial and economic evaluation. BMC Ear Nose Throat Disord 2015;15:3.

-Pierzycki RH, Edmondson-Jones M, Dawes P, Munro KJ, Moore DR, Kitterick PT: Tinnitus and sleep difficulties after cochlear implantation. Ear Hear 2016;37:e402-e408.

Punte AK, Vermeire K, Hofkens A, De Bodt M, De Ridder D, Van de Heyning P: Cochlear implantation as a durable tinnitus treatment in singlesided deafness. Cochlear Implants Int 2011;12(suppl 1):S26-S29.

Quaranta N, Wagstaff S, Baguley DM: Tinnitus and cochlear implantation. Int J Audiol 2004; 43:245-251

Ramakers GG, Smulders YE, van Zon A, Kraaijenga VJ, Stegeman I, Van Zanten GA, Grolman W: Agreement between health utility instruments in cochlear implantation. Clin Otolaryngol 2016;41:737-743.

Ramakers GG, van Zon A, Stegeman I, Grolman $\mathrm{W}$ : The effect of cochlear implantation on tinnitus in patients with bilateral hearing loss: a systematic review. Laryngoscope 2015;125: 2584-2592.

-Ramos Macías A, Falcón González JC, Manrique M, Morera C, García-Ibáñez L, Cenjor C, Coudert-Koall C, Killian M: Cochlear implants as a treatment option for unilateral hearing loss, severe tinnitus and hyperacusis. Audiol Neurootol 2015; 20(suppl 1):60-66.

Salvi R, Sun W, Ding D, Chen GD, Lobarinas E, Wang J, Radziwon K, Auerbach BD: Inner hair cell loss disrupts hearing and cochlear function leading to sensory deprivation and enhanced central auditory gain. Front Neurol 2017;8:158.

Schecklmann M, Landgrebe M, Langguth B; TRI Database Study Group: Phenotypic characteristics of hyperacusis in tinnitus. PLoS One 2014;9:e86944

Shargorodsky J, Curhan GC, Farwell WR: Prevalence and characteristics of tinnitus among US adults. Am J Med 2010;123:711-718.

Skarzynski H, Lorens A, Kruszynska M, Obrycka A, Pastuszak D, Skarzynski PH: The hearing benefit of cochlear implantation for individuals with unilateral hearing loss, but no tinnitus. Acta Otolaryngol 2017;18:1-10.
Sladen DP, Carlson ML, Dowling BP, Olund AP, Teece K, DeJong MD, Breneman A, Peterson A, Beatty CW, Neff BA, Driscoll CL: Early outcomes after cochlear implantation for adults and children with unilateral hearing loss. Laryngoscope 2016;127:1683-1688.

Tyler RS, Baker LJ: Difficulties experienced by tinnitus sufferers. J Speech Hear Disord 1983; 48:150-154.

Tyler RS, Conrad-Armes D: The determination of tinnitus loudness considering the effects of recruitment. J Speech Hear Res 1983;26:59-72.

-Tyler RS, Gogel SA, Gehringer AK: Tinnitus activities treatment. Prog Brain Res 2007;166: 425-434.

Van de Heyning P, Vermeire K, Diebl M, Nopp P, Anderson I, De Ridder D: Incapacitating unilateral tinnitus in single-sided deafness treated by cochlear implantation. Ann Otol Rhinol Laryngol 2008;117:645-652.

van Zon A, Peters JP, Stegeman I, Smit AL, Grolman W: Cochlear implantation for patients with single-sided deafness or asymmetrical hearing loss: a systematic review of the evidence. Otol Neurotol 2015;36:209-219.

van Zon A, Smulders YE, Ramakers GG, Stegeman I, Smit AL, Van Zanten GA, Stokroos RJ, Hendrice N, Free RH, Maat B, Frijns JH, Mylanus EA, Huinck WJ, Topsakal V, Tange RA, Grolman W: Effect of unilateral and simultaneous bilateral cochlear implantation on tinnitus: a prospective study. Laryngoscope 2016;126:956-961.

Vielsmeier V, Kreuzer PM, Haubner F, Steffens T, Semmler PR, Kleinjung T, Schlee W, Langguth B, Schecklmann M: Speech comprehension difficulties in chronic tinnitus and its relation to hyperacusis. Front Aging Neurosci 2016;8:293.

Wallhäusser-Franke E, Delb W, Balkenhol T, Hiller W, Hörmann K: Tinnitus-related distress and the personality characteristic resilience. Neural Plast 2014;2014:370307.

Weidt S, Delsignore A, Meyer M, Rufer M, Peter $\mathrm{N}$, Drabe N, Kleinjung T: Which tinnitus-related characteristics affect current health-related quality of life and depression? A crosssectional cohort study. Psychiatry Res 2016; 237:114-121.

Zenner HP, Delb W, Kröner-Herwig B, Jäger B, Peroz I, Hesse G, Mazurek B, Goebel G, Gerloff C, Trollmann R, Biesinger E, Seidler H, Langguth B: A multidisciplinary systematic review of the treatment for chronic idiopathic tinnitus. Eur Arch Otorhinolaryngol 2017; 274:2079-2091.
CI in Patients with Unilateral Hearing

Loss, Tinnitus, and Hyperacusis
Audiol Neurotol 2018:23:8-19

DOI: $10.1159 / 000488755$ 